\title{
Performance Evaluation of Vision-based Gait Recognition using a Very Large-scale Gait Database
}

\author{
Mayu Okumura, Haruyuki Iwama, Yasushi Makihara, Yasushi Yagi \\ Institute of Scientific and Industrial Research, Osaka University \\ \{okumura, iwama, makihara, yagi\}@am.sanken.osaka-u.ac.jp
}

\begin{abstract}
This paper describes the construction of the largest gait database in the world and its application to a statistically reliable performance evaluation of vision-based gait recognition. Whereas existing gait databases include at most an order of a hundred subjects, we construct an even larger gait database which includes 1,035 subjects $(569$ males and 466 females) with ages ranging from 2 to 94 years. Because a sufficient number of subjects for each gender and age group are included in this very large-scale gait database, we can analyze the dependence of gait recognition performance on gender or age groups. The results of GEI-based gait recognition provide several novel insights, such as the tradeoff of gait recognition performance among age groups derived from the maturity of walking ability and physical strength. Moreover, improvement in the statistical reliability of performance evaluation is shown by comparing the gait recognition results for the whole set and subsets of a hundred subjects selected randomly from the whole set.
\end{abstract}

\section{INTRODUCTION}

In modern society, there is a growing need to identify individuals in many different situations, including for surveillance and access control. For personal identification, many biometric-based authentication methods have been proposed using a wide variety of cues, such as fingerprints, irises, faces, and gait. Of these, gait identification has attracted considerable attention because it offers surveillance systems the ability to ascertain identity at a distance. Actually, automatic gait recognition on public CCTV images has been admitted as evidence in UK courts [1], and gait evidence has been used as a cue for criminal investigation in Japan.

Recently, various approaches to gait identification have been proposed. These range from model-based approaches [2] [3] [4] [5] [6] to appearance-based approaches [7] [8] [9] [10] [11] [12] [13] [14] [15]. In addition, several common gait databases have been published [16] [17] [18] [19] [20] [21] [22] [23] [24] for fair comparison of gait recognition approaches. These databases are usually constructed taking into account the following: (1) the variation in walking conditions, and (2) the number and variation of subjects.

The first consideration is important to ensure the robustness of the gait recognition algorithms, since walking conditions often differ between enrollment and test stages. For example, observation views are often inconsistent due to the positions of the CCTV cameras on the street and/or walking directions possibly being different. In addition, walking speeds can change depending on whether the person is merely taking a walk in the park or is walking to the station in a hurry, and clothing almost certainly changes depending on the season.

The second consideration is also important because the number of subjects determines the upper bound of the statistical reliability of the performance evaluation. In addition, if the database is used not only for person identification, but also gender and age estimation from gait, the variation of subjects in terms of genders and ages plays an important role in the performance evaluations of such applications.

In this paper, we focus on the second consideration and construct the largest gait database in the world, comprising more than a thousand subjects of both genders and including a wide range of ages. The largest existing gait database comprises at most an order of a hundred subjects with biased distribution of genders and ages. The proposed gait database thus enables us to evaluate gait recognition algorithms in a more statistically reliable way and to reveal how gait recognition performances differ between genders and age groups.

The outline of this paper is as follows. First, existing gait databases are briefly considered in Section II. Next, the construction of the very large-scale gait database is addressed in Section III. Several existing gait recognition approaches are examined in Section IV, with their performance evaluation using the very large-scale gait database presented in Section V. Section VI contains our conclusions and future work in the area.

\section{RELATED WORK ON EXISTING GAIT DATABASES}

The existing gait databases are summarized in Table I. First, we briefly review the walking condition variations in the databases.

The USF dataset [16] is one of the most widely used gait datasets and is composed of a gallery and 12 probe sequences under different walking conditions that include factors such as views, shoes, surfaces, baggage, and time. As the number of factors is the largest of all the existing databases, and despite the number of variations for each factor being limited to 2, the USF database is suitable for evaluating the inter-factor, instead of intra-factor, impact on gait recognition performance.

The CMU MoBo Database [19] contains image sequences of persons walking on a treadmill captured by six cameras. As the treadmill can control the walking speed and slope, the database includes gait images with speed and slope variations as well as view variations. As a result, this database is often 
used for performance evaluation for speed-invariant or viewinvariant gait recognition [11].

The CASIA dataset [23] contains the largest azimuth view variations and hence, it is used for analysis and modeling of view impact on gait recognition [25].

The OU-ISIR Gait Database [24] contains gait images with the largest speed variations ( 7 speeds: $1 \mathrm{~km} / \mathrm{h}$ interval from 2 $\mathrm{km} / \mathrm{h}$ to $7 \mathrm{~km} / \mathrm{h}$ ) and clothing variations (32 combinations at most), and therefore, it is used for evaluating speed-invariant [26] and clothing-invariant [27] gait recognition.

Next, we review the number and variation of subjects. As shown in Table I, relatively large-scale gait databases with more than a hundred subjects are limited to the following three: USF dataset [16], Soton database [20], and CASIA dataset [23]. Although these three databases provide a statistically reliable performance to some extent, the number of subjects are still not sufficient compared with other biometrics such as fingerprints and faces.

In addition, populations of genders and ages are biased in these databases; e.g., there are no children in the USF dataset, while in the CASIA dataset most of the subjects are in their twenties and thirties and the ratio of males to females is 3 to 1 . Such biases are undesirable in performance evaluation of gait-based gender and age estimation and performance comparison of gait recognition between genders and age groups.

\section{ThE LARGEST GAIT DATABASE}

\section{A. Gait measurement system}

An overview of our gait measurement system is illustrated in Fig. 1. A subject walks through the first straight course (blue arrows) and thereafter, walks through the second straight course (red arrows). The length of each course is 10.0 $\mathrm{m}$, with $3.0 \mathrm{~m}$ sections at the beginning and end regarded as acceleration and deceleration intervals, respectively. Two cameras are set along each course, approximately $4.0 \mathrm{~m}$ apart, with their observation views ranging (1) from side to rear oblique, and (2) from front oblique to side, respectively, and therefore, four sequences in total are captured for each subject. The image size and frame rate are set to 640 by 480 pixels and $30 \mathrm{fps}$, respectively.

Moreover, the background has been designed to make silhouette extraction easier. The floor and back wall are covered with green carpets and light green panels, respectively, and these are illuminated by LED lights as shown in Fig. 1. Examples of the captured images from the four cameras are shown in Fig 2.

\section{B. Data collection and statistics}

We collected the gait images, together with a demonstration of online gait personality measurement [28] for the Dive Into the Movie (DIM) project [29]. The event was held over 3 days in March 2009 and more than a thousand visitors participated in the demonstration. Each subject was requested to sign an informed consent to permit the use of the collected data for research purposes. As a result, we constructed the largest gait database in the world that includes 1,035 subjects

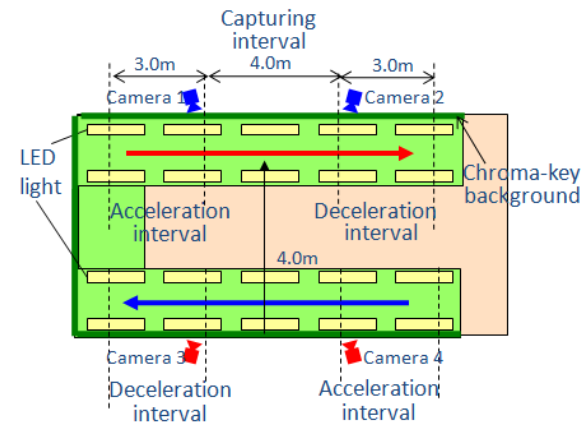

(a) Top view

Fig. 1. Gait measurement system

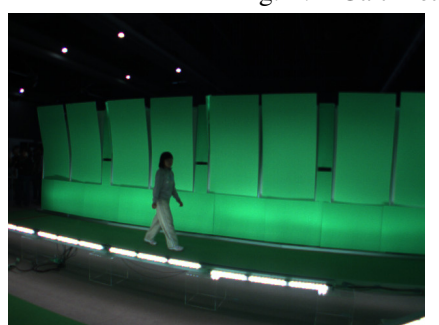

(a) Camera 1

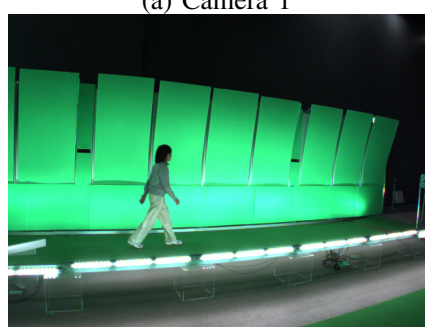

(c) Camera 3

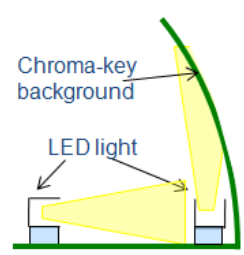

(b) Side view

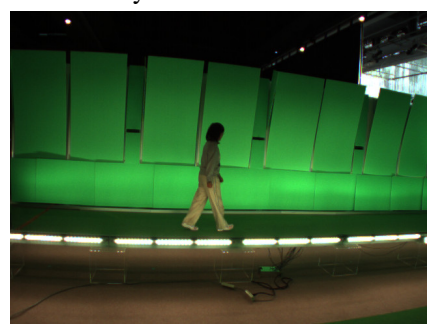

(b) Camera 2

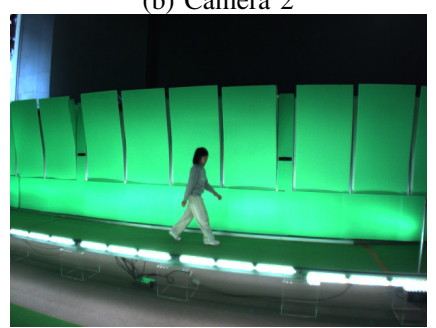

(d) Camera 4

Fig. 2. Examples of the captured images

(569 males and 466 females) with ages ranging from 2 to 94 years. Detailed statistics of the subjects are shown in Fig. 3. Compared with the existing gait databases, the advantages of this very large-scale gait database are given below.

1) The number of subjects is approximately 10 times more than in the existing large gait databases. This significantly improves the reliability of the gait recognition performance evaluation.

2) The male-to-female ratio is close to 1 . This is a desirable property for more reliable performance evaluation of gait-based gender classification and for comparison of gait recognition performance between genders.

3) The subjects' ages are widely distributed from 2 to 94 years. In particular, the number of children is comparable to the number of adults, and each 10-year interval up to 50 years contains more than a hundred subjects. This provides more statistically reliable results on gait-based age group classification and difficulty level comparison of gait recognition among age groups.

\section{GAIT FEATURE EXTRACTION}

In this section, we introduce several gait recognition algorithms used for performance evaluation.

\section{A. Extraction of Gait Silhouette Volume (GSV)}

Gait silhouette extraction is an essential preprocessing step in most of the appearance-based gait recognition approaches. 
TABLE I

EXISTING GAIT DATABASES

\begin{tabular}{l|r|l}
\hline Database & \#Subjects & Walking conditions \\
\hline \hline USF dataset [16] & 122 & 2 views, 2 shoes, 2 surfaces, baggage (w/ and w/o), time \\
UCSD database [17] & 6 & - \\
Georgia Tech database [18] & 20 & Multiple views, time, distance \\
CMU Mobo database [19] & 25 & 6 views, 2 speeds, 2 slopes, baggage (ball) \\
Soton (Southampton) database [20] & 115 & Multiple views \\
UMD (Univ. of Maryland) database [21] & 25 & 4 views \\
& 55 & 2 views \\
MIT database [22] & 24 & Multiple views \\
CASIA dataset [23] & 124 & 11 views, clothing (w/ and w/o coat), baggage (w/ and w/o) \\
OU-ISIR gait database (treadmill dataset) [24] & 34 & 7 speeds \\
& 68 & 32 clothes combination (at most) \\
\hline
\end{tabular}

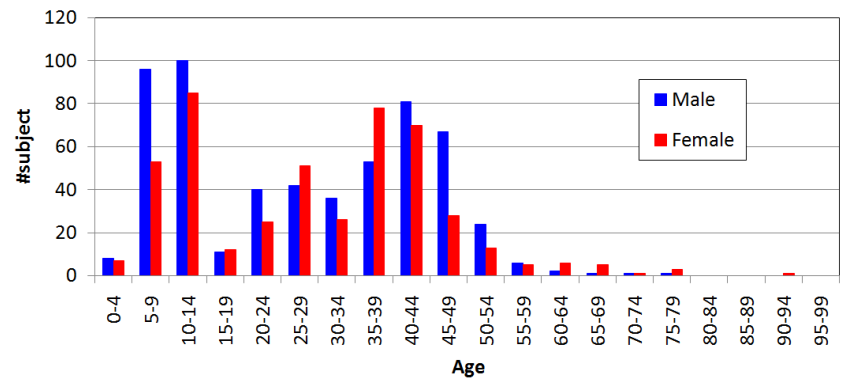

Fig. 3. Statistics of subjects' gender and age

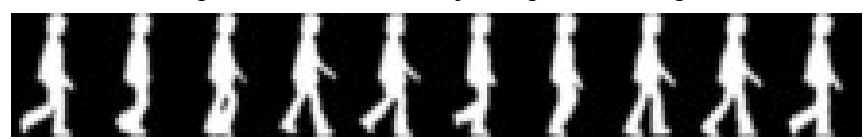

Fig. 4. An example of GSV

The current mainstream algorithm for foreground segmentation from a static background is a graph cut-based algorithm [30] in conjunction with background subtraction, which is also exploited in this work.

The next step is registration and size normalization. The height and center values of the silhouette region are obtained for each frame and the silhouette is scaled so that the height is a pre-determined size, whilst maintaining the aspect ratio. In this paper, the size is set to a height of $H=32$ pixels and a width of $W=22$ pixels. Then, each silhouette is registered such that its center corresponds to the image center. Finally, a spatio-temporal GSV is produced by stacking the silhouettes on the temporal axis. An example of a constructed GSV is shown in Fig. 4.

\section{B. Gait period detection}

Because gait is a periodic motion, the gait period $N_{\text {gait }}$ is usually regarded as a feature vector unit that needs to be detected before feature extraction. This is determined by maximizing the following normalized autocorrelation of the GSV for the temporal axis.

$$
\begin{gathered}
C(N)=\frac{\sum_{x, y} \sum_{n=0}^{T(N)} g(x, y, n) g(x, y, n+N)}{\sqrt{\sum_{x, y} \sum_{n=0}^{T(N)} g(x, y, n)^{2}} \sqrt{\sum_{x, y} \sum_{n=0}^{T(N)} g(x, y, n+N)^{2}}} \\
T(N)=N_{\text {total }}-N-1,
\end{gathered}
$$

where $g(x, y, n)$ is a silhouette value of the GSV at position $(x, y)$ of the $n$th frame, $C(N)$ is the autocorrelation for the $N$ frame shift, and $N_{\text {total }}$ is the total number of frames in the sequence. Images are captured at $30 \mathrm{fps}$ in this paper, and hence the domain of $N$ is empirically set to [20,40] frames $([0.66,1.33] \mathrm{sec})$ for natural gait periods. Other gait types such as running, brisk walking, and 'ox walking' are not within the scope of this paper.

\section{Gait trajectory matching in eigen space}

A method for gait silhouette matching with phase shift has been proposed as a baseline algorithm [16]. More sophisticated versions of this method, that is, gait trajectory matching in eigen space with linear phase warping [14] [31] are also available. In [31], PCA is first applied to reduce the dimensions of GSV. The number of reduced dimensions is determined so that the cumulative contribution ratio of eigenvalues is more than $99 \%$ in this paper. As a result, the gait silhouette and gait silhouette sequence are expressed as a point and trajectory in the PCA space, respectively.

In the matching stage, the gait trajectories of a gallery and/or probe are divided into period-unit ones and matching results on the multiple periods are statistically integrated. Finally, two types of matching units are adopted: (1) PPPCA (period-period matching in PCA space), and (2) SPPCA (sequence-period matching in PCA space).

\section{Frequency-domain feature}

In this section, a frequency-domain feature [15] is introduced as another gait feature. First, a Discrete Fourier Transform (DFT) of the temporal axis is applied to the GSV feature vector as

$$
G_{i}(x, y, k)=\sum_{n=i N_{\text {gait }}}^{(i+1) N_{\text {gait }}-1} g(x, y, n) e^{-j \omega_{0} k n},
$$

where $\omega_{0}=2 \pi / N_{\text {gait }}$ is the base angular frequency for the gait period $N_{\text {gait }}$, and $G_{i}(x, y, k)$ is the DFT of the GSV for $k$-times the base frequency. Subsequently, amplitude spectra of the DFT are calculated as

$$
A_{i}(x, y, k)=\frac{1}{N_{\text {gait }}}\left|G_{i}(x, y, k)\right|,
$$

where $A_{i}(x, y, k)$ is an amplitude spectrum for $G_{i}(x, y, k)$ normalized with the gait period $N_{\text {gait }}$. In this paper, direct current elements $(k=0)$ (averaged silhouette) and low frequency elements $(k=1,2)$ are used as frequency-domain features. Consequently, the total number of frequency elements $\left(N_{A}\right)$ is 2,112 ( 3 frequencies $\times 32$ pixels high $\times 22$ pixels wide). 

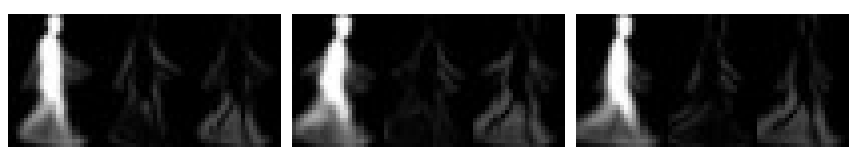

Fig. 5. Examples of frequency-domain features among three subjects. In each subject, left image indicates direct current element (GEI), middle and right images indicate 1 - and 2-times frequency elements.

Note that the only direct current element $(k=0)$ is equivalent to the averaged silhouette [32] or Gait Energy Image [33]. In the following sections, we refer to the frequency-domain feature and the Gait Energy Image as FREQ and GEI, respectively. Examples of FREQ are shown in Fig. 5.

\section{Performance eVAluation}

\section{A. Evaluation measures}

In this experiment, two side view sequences from cameras 21 and 4 are used as the gallery and probe for each subject, respectively. Recognition performance is evaluated by two measures: (1) Cumulative Match Characteristic (CMC) curve and (2) Receiver Operating Characteristic (ROC) curve [34]. The CMC curve shows the relation between identification rates and a tolerant rank in a one-to- $\mathrm{N}$ matching scenario. For example, a rank $k$ identification rate means the ratio of a probe to all other probes, where a correct gallery corresponding to the given probe occurs within a rank $k$ score. The ROC curve denotes a tradeoff curve between a False Rejection Rate (FRR) and False Acceptance Rate (FAR) when the acceptance threshold is changed by a receiver in a one-toone matching scenario. In a one-to-one matching scenario, a z-normalized distance among galleries [34] is adopted to improve the performance in this paper. Note that the CMC curve is dependent on the gallery size, whereas the ROC curve is essentially independent of the gallery size.

\section{B. Comparison of gait recognition approaches}

In this section, the recognition performance of each of the four approaches, PP-PCA, SP-PCA, GEI, and FREQ, is compared using the whole set in the largest gait database as a preliminary experiment. The CMC and ROC curves are depicted in Figs. 6 and 7, respectively. According to the results, GEI achieves the best performance of all. PP-PCA, SPPCA, and FREQ are more sensitive to motion components than GEI and therefore, their performance is possibly more severely affected by gait fluctuations between the gallery and probe. Further analysis of the decline in performance of FREQ, SP-PCA, and PP-PCA, as well as a performance comparison with other state-of-the-art approaches, is left for a future work.

\section{Impact of number of subjects}

In this section, the impact of the number of subjects is investigated. Based on a statistical analysis of ROC curves [35], the standard deviation of the FRR with a single probe for each subject is estimated as

$$
\hat{\sigma}(\hat{p})=\sqrt{\frac{\hat{p}(1-\hat{p})}{n-1}},
$$

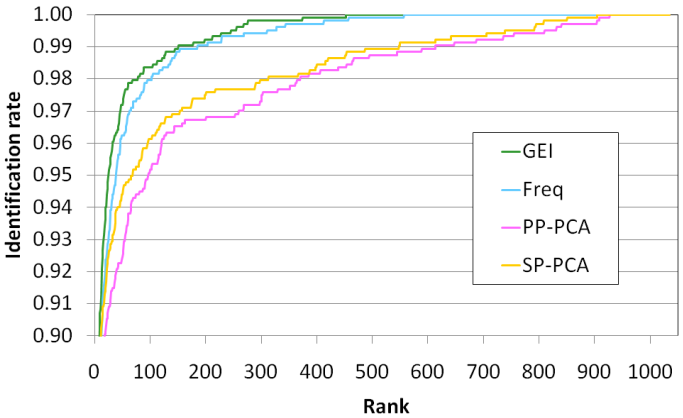

Fig. 6. CMC curve of gait recognition approaches

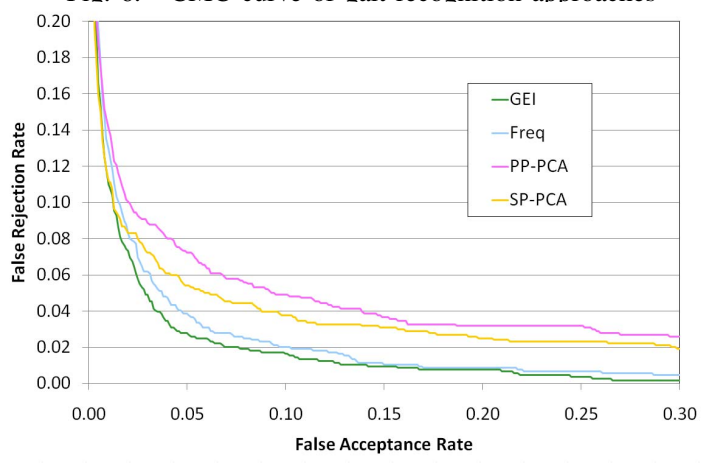

Fig. 7. ROC curve of gait recognition approaches

where $\hat{p}$ is the observed FRR and $n$ is the number of subjects. This indicates that the obtained FRR becomes more reliable as the number of subjects increases. To validate the estimation, we repeated the experiments with randomly chosen subsets with fewer subjects and compared the actual standard deviation of the performance and the estimated one from Eq. (5). First, we prepared seven subsets comprising a hundred subjects randomly chosen from the whole set and obtained seven ROC curves for GEI-based gait recognition from the experimental results. Then, we calculated the average and standard deviation of the FRR for each FAR, depicted as an averaged ROC curve (bold pink line) and standard deviation range bar (gray bar) in Fig. 8. In addition, the estimated standard deviation range from Eq. (5) is depicted as two thin pink lines. From this graph, we can see that the standard deviation ranges derived from the experimental results correspond well with those estimated from Eq. (5).

Moreover, the results for the whole set are superimposed as a bold blue line, while the standard deviation range estimated from Eq. (5) is depicted as two thin blue lines in Fig. 8. We can see that the standard deviation range is significantly narrower than that of the smaller subject subsets. For example at $4 \%$ FAR, the standard deviation is reduced from $1.9 \%$ to $0.6 \%$. This indicates that the accuracy of the performance evaluation increases three fold.

\section{Impact of gender}

In this section, we investigate how gait recognition performance differs between genders or among age groups. Our very large-scale gait database is ideally suited to this since the gender ratio is close to one as mentioned in Section III.

The ROC curve for GEI-based gait recognition performance among the 569 males is depicted as a bold blue line 


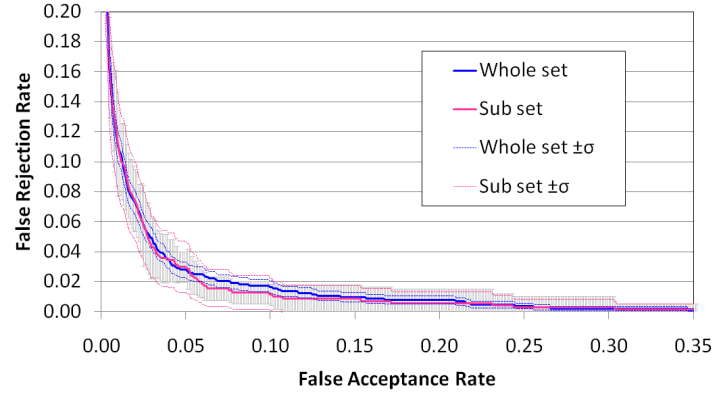

Fig. 8. ROC curves of GEI-based gait recognition with different numbers of subjects. Pink and blue stand for smaller subsets and the whole set. Bold line and two bounding thin lines mean an average $\mu$ and standard deviation range $\mu \pm \sigma$ derived from Eq. (5). Gray bars are standard deviation ranges $\mu \pm \sigma$ obtained by experiments.

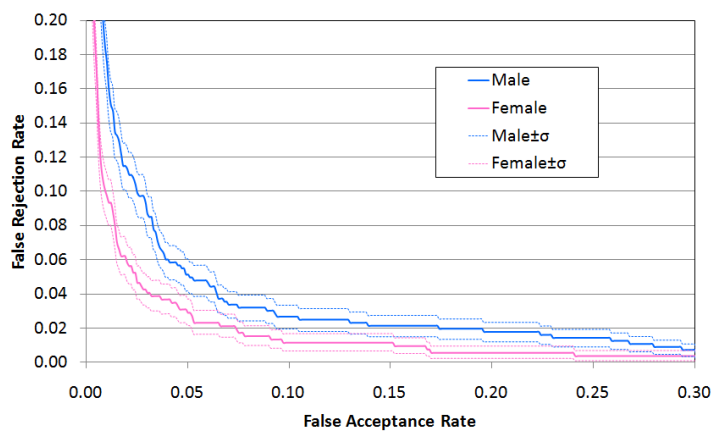

Fig. 9. ROC curves of GEI-based gait recognition between genders. Blue and pink stand for males and females respectively. Bold lines and two bounding thin lines mean an average $\mu$ and standard deviation range $\mu \pm \sigma$ derived from Eq. (5).

in Fig. 9 and that for the 466 females as a bold pink line. In addition, the standard deviation range derived from Eq. (5) is depicted as two thin lines. According to the results, the recognition performance among females is slightly better than that among males. Moreover, the distance distributions of the same subjects (true attempts) and different subjects (imposters) for males and females are depicted in Fig. 10. This result implies that the inter-subject variation of the males' gait is less than that of the females' gait and/or that the intra-subject variation, that is, the fluctuation in the males' gait is larger than that for females. In future work, we intend to thoroughly analyze the gait pattern distributions between genders.

\section{E. Impact of age groups}

Next, we show the difference in gait recognition performance among the age groups. Our very large-scale gait

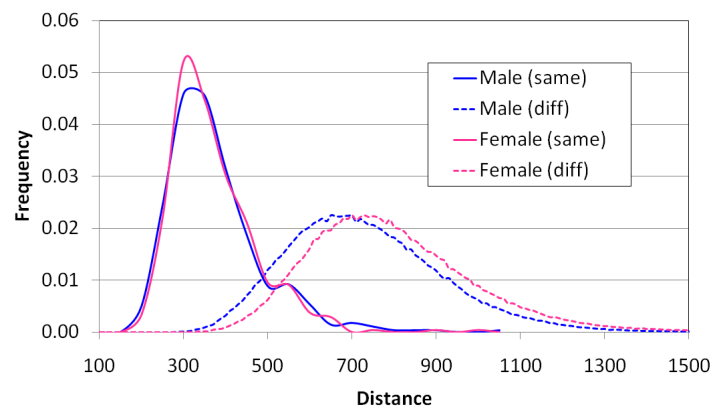

Fig. 10. Distance distribution between genders.

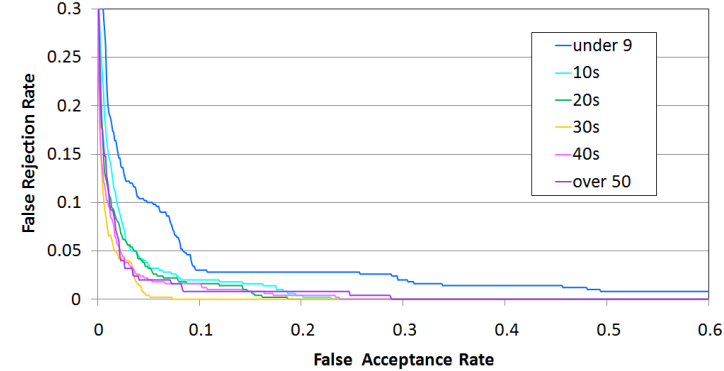

Fig. 11. ROC curves of GEI-based gait recognition for each age-group interval.

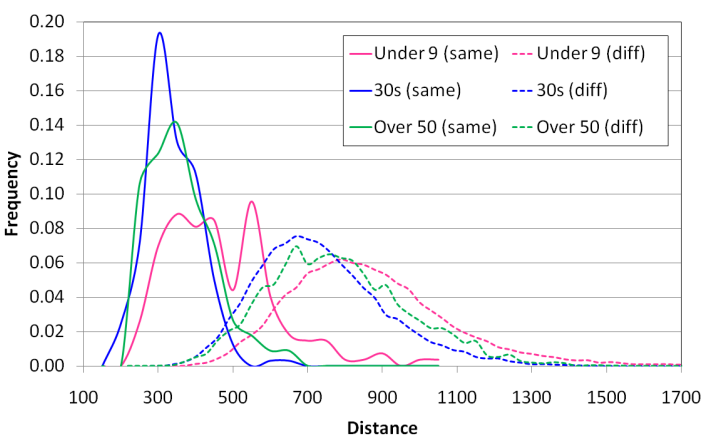

Fig. 12. Distance distribution for each age-group interval.

database is ideally suited to this purpose because the age distribution is much wider than that in existing gait databases as mentioned in Section III.

In the very large-scale gait database, each 10 -year interval up to 50 years contains more than a hundred subjects, and we randomly selected 5 subsets of a hundred subjects for each age-group interval. The interval over 50 years is treated separately as one age group because of the shortage of subjects. The averaged ROC curves for each age-group interval are depicted in Fig. 11. Moreover, the distance distributions of the same subjects (true attempts) and different subjects (imposters) for three typical age groups, under 9's, 30's, and over 50's are depicted in Fig. 12.

From the results, we can see that the gait recognition performance for the child age groups is worse than that for the other age groups, and this gradually improves with older groups up to 30 years. This result is intuitively understandable because the intra-subject gait fluctuation for children is relatively larger due to the immaturity of their walking skills. On the other hand, fluctuation in gait for adults is quite small since adults have established their own walking style; in other words, they have a fixed gait pattern.

On the other hand, the gait recognition performance for groups over 40 years old declines as the subjects become older. This degradation in performance is inferred from the fact that physical strength generally declines as the subject grows older and hence, the gait tends to fluctuate more.

Consequently, gait recognition performance of subjects in their thirties is regarded as a tradeoff between the maturity of walking ability and physical strength. Moreover, the dependence of gait fluctuation on the age group implies that gait fluctuation can be a useful cue for age classification by gait. In addition, the age group can be regarded as a so- 
called quality measure for gait recognition, which is one of the interesting future directions in this study.

\section{Conclusion}

This paper describes the construction of the world's largest gait database and a statistically reliable performance evaluation of vision-based gait recognition. The database has the following three advantages compared with existing gait databases: (1) the number of subjects is 1,035, which is approximately 10 times more than existing large-scale gait databases, (2) the male-to-female ratio is close to one, (3) the age distribution is wide, ranging from 2 to 94 years. Because sufficient numbers of subjects for each gender and age group are included in the large-scale gait database, it is possible to evaluate how gait recognition performances differ between genders and among age groups. The results of GEIbased gait recognition provide several novel insights, such as the tradeoff of gait recognition performance among age groups based on the maturity of walking ability and physical strength. Moreover, improvement in the statistical reliability of performance evaluation is shown by comparing the gait recognition results for the whole set and subsets of a hundred subjects randomly selected from the whole set.

Future work includes performance evaluation of other state-of-the-art gait recognition approaches, and gender and/or age group classification by gait using the very largescale gait database.

\section{ACKNOWLEDGMENTS}

This work was supported by Grant-in-Aid for Scientific Research(S) 21220003.

\section{REFERENCES}

[1] "How biometrics could change security," http://news.bbc.co.uk/2/hi/programmes/click_online/7702065.stm.

[2] A. Bobick and A. Johnson, "Gait recognition using static activityspecific parameters," in Proc. of IEEE Conf. on Computer Vision and Pattern Recognition, vol. 1, 2001, pp. 423-430.

[3] D. Wagg and M. Nixon, "On automated model-based extraction and analysis of gait," in Proc. of the 6th IEEE Int. Conf. on Automatic Face and Gesture Recognition, 2004, pp. 11-16.

[4] L. Wang, H. Ning, T. Tan, and W. Hu, "Fusion of static and dynamic body biometrics for gait recognition," in Proc. of the 9th International Conference on Computer Vision, vol. 2, 2003, pp. 1449-1454.

[5] R. Urtasun and P. Fua, "3d tracking for gait characterization and recognition," in Proc. of the 6th IEEE Int. Conf. on Automatic Face and Gesture Recognition, 2004, pp. 17-22.

[6] G. Zhao, G. Liu, H. Li, and M. Pietikainen, "3d gait recognition using multiple cameras," in Proc. of the 7th Int. Conf. on Automatic Face and Gesture Recognition, Apr. 2006, pp. 529-534.

[7] C. BenAbdelkader, R. Culter, H. Nanda, and L. Davis, "Eigengait: Motion-based recognition people using image self-similarity," in Proc. of Int. Conf. on Audio and Video-based Person Authentication, 2001, pp. 284-294.

[8] N. Cuntoor, A. Kale, and R. Chellappa, "Combining multiple evidences for gait recognition," in Proc. of IEEE Int. Conf. on Acoustics, Speech, and Signal Processing, vol. 3, 2003, pp. 33-36.

[9] Y. Liu, R. Collins, and Y. Tsin, "Gait sequence analysis using frieze patterns," in Proc. of the 7th European Conf. on Computer Vision, vol. 2, 2002, pp. 657-671.

[10] T. Kobayashi and N. Otsu, "Action and simultaneous multiple-person identification using cubic higher-order local auto-correlation," in Proc. of the 17th Int. Conf. on Pattern Recognition, vol. 3, Aug. 2004, pp. 741-744.
[11] Z. Liu and S. Sarkar, "Improved gait recognition by gait dynamics normalization," IEEE Transactions on Pattern Analysis and Machine Intelligence, vol. 28, no. 6, pp. 863-876, 2006.

[12] G. Veres, L. Gordon, J. Carter, and M. Nixon, "What image information is important in silhouette-based gait recognition?" in Proc. of IEEE Conf. on Computer Vision and Pattern Recognition, vol. 2, 2004, pp. $776-782$.

[13] S. Mowbray and M. Nixon, "Automatic gait recognition via fourier descriptors of deformable objects," in Proc. of IEEE Conf. on Advanced Video and Signal Based Surveillance, 2003, pp. 566-573.

[14] H. Murase and R. Sakai, "Moving object recognition in eigenspace representation: Gait analysis and lip reading," Pattern Recognition Letters, vol. 17, pp. 155-162, 1996.

[15] Y. Makihara, R. Sagawa, Y. Mukaigawa, T. Echigo, and Y. Yagi, "Gait recognition using a view transformation model in the frequency domain," in Proc. of the 9th European Conf. on Computer Vision, vol. 3, Graz, Austria, May 2006, pp. 151-163.

[16] S. Sarkar, J. Phillips, Z. Liu, I. Vega, P. Grother, and K. Bowyer, "The humanid gait challenge problem: Data sets, performance, and analysis," Trans. of Pattern Analysis and Machine Intelligence, vol. 27, no. 2, pp. 162-177, 2005.

[17] J. Little and J. Boyd, "Recognizing people by their gait: The shape of motion," Videre: Journal of Computer Vision Research, vol. 1, no. 2, pp. 1-13, 1998.

[18] R. Tanawongsuwan and A. Bobick, "A study of human gaits across different speeds," Georgia Tech, Tech. Rep., 2003.

[19] R. Gross and J. Shi, "The cmu motion of body (mobo) database," CMT, Tech. Rep., Jun. 2001.

[20] M. Nixon, J. Carter, J. Shutler, and M. Grant, "Experimental plan for automatic gait recognition," Southampton, Tech. Rep., 2001.

[21] T. Chalidabhongse, V. Kruger, and R. Chellappa, "The umd database for human identification at a distance," University of Meryland, Tech. Rep., 2001.

[22] "Mit ai database," http://www.ai.mit.edu/people/llee/HID.

[23] S. Yu, D. Tan, and T. Tan, "A framework for evaluating the effect of view angle, clothing and carrying condition on gait recognition," in Proc. of the 18th Int. Conf. on Pattern Recognition, vol. 4, Hong Kong, China, Aug. 2006, pp. 441-444.

[24] "Ou-isir gait database," http://www.am.sanken.osakau.ac.jp/GaitDB/index.html.

[25] S. Yu, D. Tan, and T. Tan, "Modelling the effect of view angle variation on appearance-based gait recognition," in Proc. of 7th Asian Conf. on Computer Vision, vol. 1, Jan. 2006, pp. 807-816.

[26] Y. Makihara, A. Tsuji, and Y. Yagi, "Silhouette transformation based on walking speed for gait identification," in Proc. of the 23rd IEEE Conf. on Computer Vision and Pattern Recognition, San Francisco, CA, USA, Jun 2010.

[27] M. A. Hossain, Y. Makihara, J. Wang, and Y. Yagi, "Clothinginvariant gait identification using part-based clothing categorization and adaptive weight control," Pattern Recognition, 2010 (to appear).

[28] M. Okumura, Y. Makihara, S. Nakamura, S. Morishima, and Y. Yagi, "The online gait measurement for the audience-participant digital entertainment," in Invited Workshop on Vision Based Human Modeling and Synthesis in Motion and Expression, Xi' an, China, Sep. 2009.

[29] "Dive into the movie project," http://www.mlab.phys.waseda.ac.jp/ DIM/home_e.html.

[30] Y. Boykov and M. Jolly, "Interactive graph cuts for optimal boundary and region segmentation of objects in n-d images," in Proc. of Int. Conf. on Computer Vision, 2001.

[31] A. Mori, Y. Makihara, and Y. Yagi, "Gait recognition using periodbased phase synchronization for low frame-rate videos," in Proc. of 20th Int. Conf. on Pattern Recognition, Istanbul, Turkey, Aug. 2010.

[32] Z. Liu and S. Sarkar, "Simplest representation yet for gait recognition: Averaged silhouette," in Proc. of the 17th Int. Conf. on Pattern Recognition, vol. 1, Aug. 2004, pp. 211-214.

[33] J. Han and B. Bhanu, "Individual recognition using gait energy image," Trans. on Pattern Analysis and Machine Intelligence, vol. 28, no. 2, pp. 316- 322, 2006.

[34] P. Phillips, D. Blackburn, M. Bone, P. Grother, R. Micheals, and E. Tabassi, "Face recogntion vendor test," http://www.frvt.org,, 2002.

[35] G. SNEDECOR and W. COCHRAN, Statitical methods. Iowa State University Press, 1967. 